\title{
BMJ Open Microsimulation model for the health economic evaluation of osteoporosis interventions: study protocol
}

\author{
Lei Si, ${ }^{1,2}$ John A Eisman, ${ }^{3,4,5}$ Tania Winzenberg, ${ }^{2}$ Kerrie M Sanders, ${ }^{6}$ \\ Jacqueline R Center, ${ }^{3,5}$ Tuan V Nguyen, ${ }^{3,5,7}$ Andrew J Palmer ${ }^{2,8}$
}

To cite: Si L, Eisman JA, Winzenberg T, et al. Microsimulation model for the health economic evaluation of osteoporosis interventions: study protocol. BMJ Open 2019;9:e028365. doi:10.1136/ bmjopen-2018-028365

- Prepublication history for this paper is available online. To view these files, please visit the journal online (http://dx.doi. org/10.1136/bmjopen-2018028365).

Received 5 December 2018 Revised 16 December 2018 Accepted 17 December 2018

Check for updates

(C) Author(s) (or their employer(s)) 2019. Re-use permitted under CC BY-NC. No commercial re-use. See rights and permissions. Published by BMJ.

For numbered affiliations see end of article.

Correspondence to Professor Andrew J Palmer; andrew.palmer@utas.edu.au

\section{ABSTRACT}

Introduction 0steoporosis is a systemic skeletal disease that is characterised by reduced bone strength and increased fracture risk. Osteoporosis-related fractures impose enormous disease and economic burden to the society. Although many treatments and health interventions are proven effective to prevent fractures, health economic evaluation adds evidence to their economic merits. Computer simulation modelling is a useful approach to extrapolate clinical and economic outcomes from clinical trials and it is increasingly used in health economic evaluation. Many osteoporosis health economic models have been developed in the past decades; however, they are limited to academic use and there are no publicly accessible health economic models of osteoporosis.

Methods and analysis We will develop the Australian osteoporosis health economic model based on our previously published microsimulation model of osteoporosis in the Chinese population. The development of the model will follow the recommendations for the conduct of economic evaluations in osteoporosis by the European Society for Clinical and Economic Aspects of Osteoporosis, Osteoarthritis and Musculoskeletal Diseases and the US branch of the International Osteoporosis Foundation. The model will be a state-transition semiMarkov model with memory. Clinical parameters in the model will be mainly obtained from the Dubbo Osteoporosis Epidemiology Study and the health economic parameters will be collected from the Australian arm of the International Costs and Utilities Related to Osteoporotic Fractures Study. Model transparency and validates will be tested using the recommendations from Good Research Practices in Modelling Task Forces. The model will be used in economic evaluations of osteoporosis interventions including pharmaceutical treatments and primary care interventions. A user-friendly graphical user interface will be developed, which will connect the user to the calculation engine and the results will be generated. The user interface will facilitate the use of our model by people in different sectors.

Ethics and dissemination No ethical approval is needed for this study. Results of the model validation and future economic evaluation studies will be submitted to journals. The user interface of the health economic model will be publicly available online accompanied with a user manual.

\section{Strengths and limitations of this study}

- We will develop a state-transition microsimulation for health economic evaluation of osteoporosis interventions.

- The model will be based on the Australian setting and will be adapted and adaptable to other settings.

- The model will be publicly available with a web interface to allow access for future users to evaluate new osteoporosis interventions of interest in their own settings.

- The model will be developed following the consensus statement endorsed by the Scientific Advisory Board of Economic Aspects of Osteoporosis, Osteoarthritis and Musculoskeletal Diseases, the Committee of Scientific Advisors and the Committee of National Societies of International Osteoporosis Foundation, the US National Osteoporosis Foundation and Osteoporosis Canada.

\section{INTRODUCTION}

Osteoporosis is a common disease that is usually silent until a fracture occurs. Fracture is a global health concern particularly in women. Australian women aged 60 years were estimated to have a residual lifetime fracture risk of $44 \%$ and the risk for Chinese women was approximately 33\%. ${ }^{12}$ Despite a lower residual lifetime risk of having a fracture in men, the overall mortality HR in men after a fracture was higher than women. ${ }^{3}$ For those who survived fractures, quality of life (QoL) deteriorated dramatically up to 6 months after fracture and the loss of QoL persisted in the remaining lifetime. ${ }^{4}$ With an accelerated ageing global population, the number of fractures will increase in the coming decades. 56

Bone strength is mainly reflected by bone mineral density (BMD). Individuals with reduced $\mathrm{BMD}$ are at the greatest risk of osteoporotic fracture. The assessment of fracture risk is largely based on clinical risk factors with and without BMD. ${ }^{7}$ With the 
predicted fracture risk, treatment decisions can be made following primary or secondary fracture prevention. ${ }^{89}$

Numerous therapeutic regimes, including pharmaceutical interventions, have been developed to treat osteoporotic patients by improving their BMD. While treatment of osteoporosis has proven effective in preventing future fractures, the associated cost must be considered against the costs of not preventing fractures. In 2011/2012, the Australian government spent over 179 million Australian dollars in pharmaceuticals for osteoporosis management. ${ }^{10}$ Similar costs of osteoporosis medications have been noted elsewhere. ${ }^{611} 12$ With a range of osteoporosis treatments available in the community, some governments employ health economic evaluation in treatment reimbursement policy making, such as the Pharmaceutical Benefits Advisory Committee (PBAC) in Australia and the National Institute for Health and Care Excellence (NICE) in the UK. These government agencies conduct health economic evaluations of osteoporosis treatments to select those that represent good value for money.

Health economic evaluation of osteoporosis interventions is commonly conducted using disease modelling approaches ${ }^{13}$ such as Markov cohort models and individual-level state-transition models. ${ }^{14-16}$ Health economic modelling using different sources of information is able to capture long-term cost and health benefits of osteoporosis interventions. Health economic analyses of osteoporosis interventions may be limited due to the validity, transparency and accessibility of health economic models that are used in the analyses. Making health economic models transparent via thorough documentation and making them available for public use are potential solutions to minimise these limitations; moreover, these solutions provide opportunities to update existing models with newly available epidemiological and health economic data as they become available. ${ }^{17}$ To date, there is no publicly accessible health economic model of osteoporosis.

\section{OBJECTIVE}

We aim to develop a publicly available health economic model to evaluate the cost-effectiveness of osteoporosis interventions. The model will be based on the Australian setting and will be adapted and adaptable to other settings. Moreover, we will develop a web interface to allow access for future users to evaluate new osteoporosis interventions of interest in their own settings.

\section{METHODS AND DESIGN}

\section{Model structure and parameters}

We have published a microsimulation model of osteoporosis in the Chinese population and will develop the Australian model using the existing model structure. ${ }^{18}$ The model will incorporate Australian fracture risk equations for estimating absolute risk of osteoporosis fractures based on patients' characteristics (eg, age, sex, family history, BMD, history of fractures and falls as well

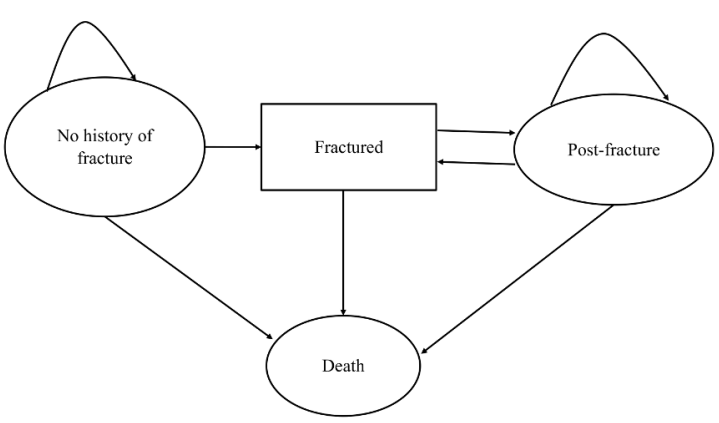

Figure 1 Structure of the osteoporosis state-transition model. Simulated patients transit in the model following the arrow direction. Simulation is concluded when all simulated patients transit to the 'death' state. Reprinted by permission from Springer Nature Customer Service Centre GmbH: Springer Nature. ${ }^{18}$

as comorbidities), and time-varying factors such as exposure to primary and/or secondary fracture prevention therapy and patients' accumulated history of fractures. These equations will form the basis of the simulation model for estimating outcomes for Australian patients with osteoporosis.

The development of our osteoporosis health economic model will follow the recently published modelling guideline in osteoporosis. ${ }^{19}$ Simulations will be based on a state-transition individual patient simulation model (figure 1). Simulated patients in the model are either alive or dead based on annual transitions. Outcomes will be analysed over patients' lifetimes but time horizons may be varied according to the study of interest. Simulated patients will transit in the model until they are absorbed in the 'death' state.

Fragility facture is a key outcome of osteoporosis and can occur in hip (proximal femur), vertebrae (spine), wrist (distal radius) and other non-hip non-vertebral sites such as humerus, pelvis, ribs and shoulder. Simulated patients can have multiple fractures at different fracture sites. Fracture probabilities will be simulated from one of three risk engines. First, for countries that report fracture incidence rates, fracture probabilities will be calculated from incidence rates using the following equation:

$$
p=1-\exp (-r)
$$

where $\mathrm{p}$ is the probability and $\mathrm{r}$ is the annual fracture incidence rate by sex, fracture site and BMD level. In the model for the Australian population, annual fracture incidence rates by sex, fracture site and BMD level will be obtained from the Dubbo Osteoporosis Epidemiology Study. ${ }^{20}$ For countries without reported fracture incidence rates, users will be able to choose the formula for calculation of annual fracture probabilities. Ten-year risk for hip and all fragility fractures are predicted by the FRAX or Garvan Fracture Risk Calculator (GARVAN-FRC). ${ }^{21-24}$ The annual probability of having a fracture will be calculated as: 


$$
p=1-\exp \left(\frac{\ln (1-\mathrm{P})}{\mathrm{t}}\right)
$$

where $\mathrm{P}$ is the cumulative risk of having a fracture over a period of $t$ years. For example, for a given set of clinical risk factors, a group of patients is estimated to have a 5\% 10-year probability of having a hip fracture. In this scenario, the annual probability of having a hip fracture is calculated as $0.51 \%$.

Simulated patients could stay without any fracture for the entire life (represented as 'no history of fracture'), sustain a fracture (represented as 'fractured'), stay in postfracture state (represented as 'postfracture') or sustain another fracture (represented as 'fractured'). In the case of death, Australian life tables combined with fracture site-specific relative risks adjusted by time since fracture will be used to calculate the probability of death during the course of the simulation. ${ }^{3}$

The model will primarily take the societal perspective, which is a broader perspective accounting for all stakeholders. In the Australian model, we will use data from the Australian arm of the International Costs and Utilities Related to Osteoporotic Fractures Study (AusICUROS) to calculate the costs of direct health and non-healthcare utilisation. ${ }^{10}$ Specifically, direct healthcare utilisation included hospitalisation, ambulance, imaging, medical services, pharmaceuticals and supplements, non-admitted, subacute/rehabilitation and community-based services including GP and physiotherapy services. Direct non-healthcare utilisation included residential care, meals on wheels and other community services. ${ }^{10}$ Relevant unit costs of the above healthcare utilisation will be taken from Pharmaceutical Benefit Scheme (PBS), Medical Benefit
Scheme (MBS) and other government reporting system such as the Australian-refined diagnosis-related groups (AR-DRGs). In addition, we will include indirect costs of productivity loss. While societal perspective will be used primarily, the cost data will be categorised to be adapted for a different perspective for future use. For example, when evaluating the cost-effectiveness of an osteoporosis medication for listing on the PBS, only costs borne by the Australian government will be included.

While the model can estimate the life expectancy, it will primarily use quality-adjusted life years (QALYs) to quantify health outcomes, since this is the most commonly used metric in health economic evaluations. The QALY, which adjusts life expectancy by the degree of morbidity, is usually measured on a health state utility (HSU) scale where zero represents death and one represents full health in each year of life. HSUs for each level of disease severity will be incorporated from the AusICUROS and an international meta-analysis of HSUV changes before and after osteoporosis-related fractures. ${ }^{1025}$

The overview of the osteoporosis model algorithm is illustrated in figure 2.

\section{Microsimulation}

The Markov model is commonly used in chronic disease modelling to facilitate the fact that patients can transit to disease states repetitively during simulation. However, the use of Markov modelling is limited to the Markov assumption, which relates to the fact that the future transitions are not dependent on previous states. ${ }^{26}$ In osteoporosis epidemiology, patients' characteristics play an important role in transition between disease states. For example, the relative risk of having a subsequent fracture for patients

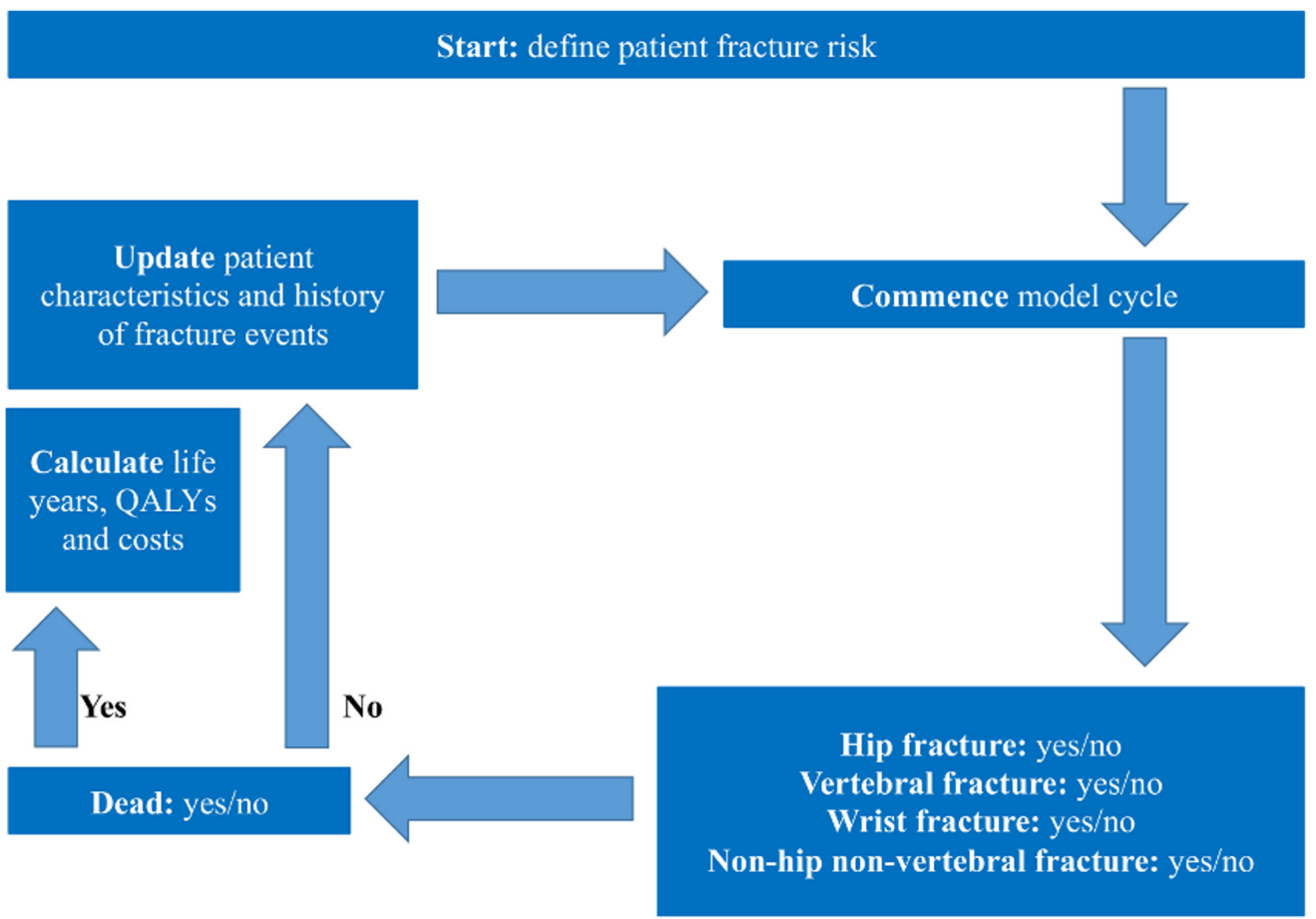

Figure 2 Overview of osteoporosis model algorithm. QALYs, quality-adjusted life years. 
with a prior fracture was higher and varied by sex, fracture site and BMD level. ${ }^{27}$ Likewise, mortality was also higher after fracture. ${ }^{3}$ Therefore, we will use an individual-level state-transition model to incorporate the memory of events occurring for simulated patients in the model. Tracker variables will be used to record events of interest during simulation. In our model, we will define the following tracker variables to represent the heterogeneity of simulated patients: 'site of each fracture', 'number of fracture by fracture site', 'time of the first fracture', 'time since the last fracture' and 'time after treatment onset'.

\section{Management with uncertainties and reporting uncertain analyses}

Four types of uncertainties are commonly considered in health economic modelling studies, namely stochastic uncertainty, parameter uncertainty, heterogeneity and structural uncertainty. ${ }^{28}$ These uncertainities will be dealt with during the development of the model and analyses. For stochastic uncertainty, sufficient number of first-order Monte Carlo simulations will be conducted. For parameter uncertainties, CI of parameters along with point estimates will be included in the model. For instance, gamma or log normal distribution for cost data, log normal distribution for relative risks or hazard ratios will be used. ${ }^{29}$ Both deterministic and probabilistic sensitivity analyses will be conducted to report uncertainties. One-way sensitivity analysis will be performed to evaluate how results change by changing the value of individual parameters and the results will be presented in tornado diagrams. Probabilistic sensitivity analysis (PSA) will be conducted by simultaneous sampling of all parameters that are defined by distributions. Cost-effectiveness acceptability curves (CEAC) and distribution of net monetary or net health benefits will be presented to illustrate the cost-effectiveness decisions given a range of willingness-to-pay (WTP) thresholds. Handling uncertainties and reporting uncertainty analyses will be conducted in line with the Good Research Practices in Modelling Task Force-6. ${ }^{28}$

\section{Model transparency and validation}

Cost-effectiveness of health interventions from modelling studies informs policy decision makers when they prioritise funding for scarce healthcare resources. Therefore, the validity and transparency of a health economic model are keys in assisting policy decision making. Transparency of health economic models is subject to how well the model is documented including its structure, parameters, equations and key assumptions used in the model. Validity refers to how well the model represents the real world. ${ }^{30}$ To ensure our model will be developed following recommendations from the Good Research Practices in Modelling Task Force- $7,{ }^{30}$ we will make the non-technical documentation available following the example of the previous work in diabetes from our team. ${ }^{31}$ There are five aspects of validity, namely face validity, internal and external validities, cross validity and predictive validity. ${ }^{30}$
In our study, we will primarily deal with face, internal and external validities of our osteoporosis model.

For face validity, the clinicians in our team (JAE, TW, JRC and AJP) will evaluate whether the model presents the osteoporosis clinical pathway and the current osteoporosis management algorithms in Australia. For internal and external validity, we will conduct goodness-of-fit analyses on the model results against values used in developing the model (ie, internal validity) and that from literature (external validity). In particular, we will compare the following values: annual fracture rates by age and sex; life expectancies by age and sex; residual lifetime fracture risks by age, sex and BMD level. Goodness-of-fit analysis will be conducted using linear regression. The slope of the regression line along with the R-squared of the regression line and the root mean square error (RMSE) will be used to assess the goodness-of-fit of the model predictions and that from the literature. ${ }^{32}$ In addition, the Bland-Altman statistics will be reported to evaluate the agreement between model results and real-world observed results. ${ }^{33}$

\section{Patient and public involvement}

Patients and public will not be involved in this study.

\section{APPLICATION OF THE MODEL}

The first focus of the model application will be to quantify the cost-effectiveness of all first-line osteoporosis secondary prevention fracture medications currently reimbursed by the PBS in order to identify the most cost-effective medications and to further identify currently reimbursed medications that are not cost-effective indicating the need for disinvestment in these medications. ${ }^{34}$ The second focus will be to identify the most cost-effective osteoporosis screening and treatment strategy in Australia, as has been performed in China. ${ }^{35}$

The model will be used to evaluate the costs and incremental cost-effectiveness of alternative new interventions for primary and/or secondary fracture prevention. Modelling is usually required in economic evaluations of therapies for osteoporosis fracture prevention, as most trials end before long-term outcomes are known.

Evaluating alternative management strategies will comprise the following steps:

i. Development of the scenario to be simulated. This involves choosing an intervention or management guideline for evaluation. Evidence must be available on its effect on fracture risks and synthesis undertaken to provide inputs for the simulation model. In the case of this analysis, the intervention to be evaluated may include denosumab, bisphosphonates and other commonly used medical treatments presently available to confirm cost-effectiveness, or to identify for disinvestment if no longer found to be cost-effective; and any new medications not yet reimbursed under the MBS.

ii. Simulating long-term outcomes and costs. This will involve carrying out two simulations: in the first, the 
target population is assumed to receive standard of care and in the second, the target population is assumed to receive standard of care plus the alternative treatment;

iii. Estimation of the incremental cost-effectiveness ratio. Results from the simulation model will be combined with other information (eg, the cost of treatment) in order to undertake an incremental cost-effectiveness analysis in which the net cost (ie, treatment costs minus savings due to fractures avoided) is divided by the net effectiveness (QALYs gained).

To ensure that the work undertaken has relevance to policy, we intend to hold a workshop on osteoporosis modelling in Australia during the course of this project. The aim of the workshop will be to obtain the opinions of key stakeholders (eg, officers from the Department of Health \& Aging, representatives from Osteoporosis Australia (patients' perspectives), clinicians from the Royal Australian College of General Practitioners and representatives from pharmaceutical companies) to assist in prioritising the work programme.

\section{Model interface and availability}

We will capitalise on our team member AJP's extensive experience in his previous CORE Diabetes Model to ensure similar successful translation of the model into general use. ${ }^{36}$ A user-friendly graphical user interface will be developed, which will connect the user to the calculation engine and the results are generated. Input data, such as cohort characteristics, key cost and utility parameters, and key treatment characteristics (costs and effects) will be accessible and editable by the user. They will be stored in a structured query language (SQL) database. Results generated by the model will also be written to and stored in a database for easy future access by the user. A user instruction manual and help files will be developed.

Like the CORE Diabetes Model, the osteoporosis model will be made available to university or other public sector research groups at no cost, while those seeking to use it for commercial use (eg, such as pharmaceutical companies preparing a regulatory submission) will pay a license fee that will support maintenance and further development of the web-based model.

\section{Author affiliations}

${ }^{1}$ The George Institute for Global Health, UNSW Sydney, Sydney, New South Wales, Australia

${ }^{2}$ Menzies Institute for Medical Research, University of Tasmania, Hobart, Tasmania, Australia

${ }^{3}$ Bone Biology Division, Garvan Institute of Medical Research, Sydney, New South Wales, Australia

${ }^{4}$ School of Medicine Sydney, University of Notre Dame Australia, Sydney, New South Wales, Australia

${ }^{5}$ St Vincent's Clinical School, UNSW Sydney, Sydney, New South Wales, Australia ${ }^{6}$ Department of Medicine- Western Health, Melbourne Medical School, The University of Melbourne, Melbourne, Victoria, Australia

${ }^{7}$ School of Biomedical Engineering, University of Technology Sydney, Sydney, New South Wales, Australia

${ }^{8}$ Centre for Health Policy, School of Population and Global Health, The University of Melbourne, Melbourne, Victoria, Australia
Contributors LS and AJP contributed to the initial idea of the study. LS, JE, TW, KMS, JRC, TN and AJP contributed to the design of the work. LS drafted the manuscript. LS, JAE, TW, KMS, JRC, TVN and AJP contributed critical review for the intellectual content and approved the final version of the manuscript submitted for publication.

Funding This study is supported by a National Health and Medical Research Council (NHMRC) project grant (APP1127827). LS is a recipient of the NHMRC Early Career Fellowship (GNT1139826). The contents of this publication is solely the responsibility of the authors and does not necessarily represent the official views of the NHMRC.

Competing interests None declared.

Patient consent for publication Not required.

Provenance and peer review Not commissioned; peer reviewed for ethical and funding approval prior to submission.

Open access This is an open access article distributed in accordance with the Creative Commons Attribution Non Commercial (CC BY-NC 4.0) license, which permits others to distribute, remix, adapt, build upon this work non-commercially, and license their derivative works on different terms, provided the original work is properly cited, appropriate credit is given, any changes made indicated, and the use is non-commercial. See: http://creativecommons.org/licenses/by-nc/4.0/.

\section{REFERENCES}

1. Nguyen ND, Ahlborg HG, Center JR, et al. Residual lifetime risk of fractures in women and men. J Bone Miner Res 2007;22:781-8.

2. Si L, Winzenberg TM, Chen M, et al. Residual lifetime and 10 year absolute risks of osteoporotic fractures in Chinese men and women. Curr Med Res Opin 2015;31:1149-56.

3. Bliuc D, Nguyen ND, Milch VE, et al. Mortality risk associated with low-trauma osteoporotic fracture and subsequent fracture in men and women. JAMA 2009;301:513-21.

4. Svedbom A, Borgstöm F, Hernlund E, et al. Quality of life for up to 18 months after low-energy hip, vertebral, and distal forearm fractures-results from the ICUROS. Osteoporos Int 2018;29:557-66.

5. Odén A, McCloskey EV, Kanis JA, et al. Burden of high fracture probability worldwide: secular increases 2010-2040. Osteoporos Int 2015;26:2243-8.

6. Si L, Winzenberg TM, Jiang Q, et al. Projection of osteoporosisrelated fractures and costs in China: 2010-2050. Osteoporos Int 2015;26:1929-37.

7. Leslie WD, Lix LM. Comparison between various fracture risk assessment tools. Osteoporos Int 2014;25:1-21.

8. Cosman F, de Beur SJ, LeBoff MS, et al. Clinician's Guide to Prevention and Treatment of Osteoporosis. Osteoporos Int 2014;25:2359-81.

9. Papaioannou A, Morin S, Cheung AM, et al. 2010 clinical practice guidelines for the diagnosis and management of osteoporosis in Canada: summary. CMAJ 2010;182:1864-73.

10. Watts JJ, Abimanyi-Ochom J, Sanders KM. Osteoporosis costing all Australian: a new burden of disease analysis-2012 to 2022, 2013.

11. Hernlund E, Svedbom A, Ivergård M, et al. Osteoporosis in the European Union: medical management, epidemiology and economic burden. A report prepared in collaboration with the International Osteoporosis Foundation (IOF) and the European Federation of Pharmaceutical Industry Associations (EFPIA). Arch Osteoporos 2013;8:136.

12. Brown $P$, McNeill R, Leung W, et al. Current and future economic burden of osteoporosis in New Zealand. Appl Health Econ Health Policy 2011;9:111-23.

13. Si L, Winzenberg TM, Palmer AJ. A systematic review of models used in cost-effectiveness analyses of preventing osteoporotic fractures. Osteoporos Int 2014;25:51-60.

14. Majumdar SR, Lier DA, Leslie WD. Cost-effectiveness of two inexpensive postfracture osteoporosis interventions: results of a randomized trial. J Clin Endocrinol Metab 2013;98:1991-2000.

15. Bleibler F, Rapp K, Jaensch A, et al. Expected lifetime numbers and costs of fractures in postmenopausal women with and without osteoporosis in Germany: a discrete event simulation model. BMC Health Serv Res 2014;14:284.

16. Si L, Winzenberg TM, Chen M, et al. Screening for osteoporosis in Chinese post-menopausal women. Osteoporosis International 2016;27:2259-69.

17. Dunlop WCN, Mason N, Kenworthy J, et al. Benefits, challenges and potential strategies of open source health economic models. Pharmacoeconomics 2017;35:125-8. 
18. Si L, Winzenberg TM, Jiang $Q$, et al. Screening for and treatment of osteoporosis: construction and validation of a state-transition microsimulation cost-effectiveness model. Osteoporos Int 2015;26:1477-89.

19. Hiligsmann M, Reginster JY, Tosteson ANA, et al. Recommendations for the conduct of economic evaluations in osteoporosis: outcomes of an experts' consensus meeting organized by the European Society for Clinical and Economic Aspects of Osteoporosis, Osteoarthritis and Musculoskeletal Diseases (ESCEO) and the US branch of the International Osteoporosis Foundation. Osteoporos Int 2019;30.

20. Center JR. Fracture burden: What two and a half decades of Dubbo Osteoporosis Epidemiology Study data reveal about clinical outcomes of osteoporosis. Curr Osteoporos Rep 2017;15:88-95.

21. The University of Sheffield. Fracture risk assessment tool. 2008. https://www.sheffield.ac.uk/FRAX/.

22. Garvan Institute of Medical Research. Fracture risk calculator. https:// www.garvan.org.au/promotions/bone-fracture-risk/calculator/

23. Nguyen ND, Frost SA, Center JR, et al. Development of a nomogram for individualizing hip fracture risk in men and women. Osteoporos Int 2007;18:1109-17.

24. Nguyen ND, Frost SA, Center JR, et al. Development of prognostic nomograms for individualizing 5-year and 10-year fracture risks. Osteoporos Int 2008;19:1431-44.

25. Si L, Winzenberg TM, de Graaff B, et al. A systematic review and meta-analysis of utility-based quality of life for osteoporosis-related conditions. Osteoporos Int 2014;25:1987-97.

26. Briggs A, Sculpher M, Claxton K. Decision modelling for health economic evaluation: OUP Oxford, 2006.

27. Bliuc D, Alarkawi D, Nguyen TV, et al. Risk of subsequent fractures and mortality in elderly women and men with fragility fractures with and without osteoporotic bone density: The Dubbo Osteoporosis Epidemiology Study. J Bone Miner Res 2015;30:637-46.

28. Briggs $\mathrm{AH}$, Weinstein MC, Fenwick EA, et al. Model parameter estimation and uncertainty: a report of the ISPOR-SMDM Modeling Good Research Practices Task Force-6. Value Health 2012;15:835-42.

29. Gray AM, Clarke PM, Wolstenholme JL, et al; Applied methods of cost-effectiveness analysis in healthcare: Oxford University Press, 2011.

30. Eddy DM, Hollingworth W, Caro JJ, et al. Model transparency and validation: a report of the ISPOR-SMDM modeling good research practices task force-7. Value Health 2012;15:843-50.

31. Palmer AJ, Roze S, Valentine WJ, et al. The CORE Diabetes Model: Projecting long-term clinical outcomes, costs and cost-effectiveness of interventions in diabetes mellitus (types 1 and 2) to support clinical and reimbursement decision-making. Curr Med Res Opin 2004;20 Suppl 1(Suppl 1):S5-S26.

32. Browne MW, Cudeck R. Alternative ways of assessing model fit. Sociol Methods Res 1992;21:230-58.

33. Bland JM, Altman DG. Statistical methods for assessing agreement between two methods of clinical measurement. Lancet 1986;1:307-10.

34. Kanis JA, Brazier JE, Stevenson M, et al. Treatment of established osteoporosis: a systematic review and cost-utility analysis. Health Technol Assess 2002;6:1-146.

35. Si L, Winzenberg TM, Chen M, et al. Screening for osteoporosis in Chinese post-menopausal women: a health economic modelling study. Osteoporos Int 2016;27:2259-69.

36. Palmer AJ, Roze S, Valentine WJ, et al. Validation of the CORE Diabetes Model against epidemiological and clinical studies. Curr Med Res Opin 2004;20 Suppl 1:S27-S40. 